\title{
Semiclassical quantization condition for magnetic energy levels of electrons in metals with band-contact lines
}

\author{
G. P. Mikitik and Yu. V. Sharlai \\ B. Verkin Institute for Low Temperature Physics and Engineering, \\ National Academy of Sciences of Ukraine, 47 Lenin ave., 310164, Kharkov, Ukraine \\ E-mail: mikitik@ilt.kharkov.ua \\ Received August 31, 1998
}

\begin{abstract}
We refine the well-known quantization condition for magnetic energy levels of a semiclassical electron. The refined condition results in the energy shift of the levels when in $\mathbf{k}$ space the closed electron orbit links to the band-contact line (i.e., surrounds it). This effect is closely analogous to that of Aharonov-Bohm provided the band-contact line plays the role of the infinitely thin «solenoid» with the fixed «magnetic flux». The predicted shift must manifest itself in oscillation phenomena.
\end{abstract}

PACS: 71.70.Di, 03.65.Bz

It is common knowledge that the degeneracy of electron energy bands in a metal can occur along symmetry axes of its Brillouin zone. In addition, as was shown by Herring [1], there are lines of an accidental contact between the bands in crystals. The term «accidental» means that the degeneracy of electron states is not caused by their symmetry. Such band-contact lines is likely to exist in many metals. This statement is easily understood when one takes into account Herring's result obtained for the case of a crystal with a center of inversion: if there is a point of contact between two energy bands in an axis of symmetry of the Brillouin zone, and the interband matrix element of the velocity operator is nonzero at this point, then a band-contact line has to pass through the point. Most metals have a center of inversion (and only such ones are considered below). Moreover, it is known (see, e.g., Ref. 2) that bands in many metals intersect at points on axes of symmetry. As for the matrix element of the velocity operator, the necessary information on it follows from the irreducible representations of the intersecting bands. The simple analysis of literature data shows that the lines of the accidental contact must exist, for example, in $\mathrm{Be}, \mathrm{Mg}, \mathrm{Zn}, \mathrm{Cd}, \mathrm{Al}$ and other metals (see Fig. $1, a, b)$. Strictly speaking, any degeneracy of bands along a line of the Brillouin zone (excluding spin degeneracy) is lifted by the spin-orbit interac- tion. However, if this interaction is weak, the bands still approach each other, and the energy gap between them is small near that line in which the contact of the bands would take place if we ignored the interaction. Moreover, the inclusion of the weak spin-orbit coupling leaves the magnetic energy levels practically unchanged [3]. For this reason, to elucidate the heart of the matter, we completely neglect the spin-orbit interaction and spin of an electron in the subsequent discussion.

Semiclassical magnetic energy levels of electrons provide the basis for the analysis of many physical phenomena in metals $[4,5]$. These levels were studied in a number of papers [6-17]. It was established that a semiclassical electron orbit in the space of wave vectors, $\mathbf{k}$, is the intersection of the constantenergy surface, $\varepsilon=\mathrm{const}$, with the plane, $k_{z}=$ $=$ const, where $z$ is the direction of the magnetic field $\mathbf{H}$. In the case of closed orbits the quantization condition for magnetic energy levels looks like

$$
S\left(\varepsilon, k_{z}\right)=(2 \pi|e| H / \hbar c)(n+\gamma),
$$

where $S$ is the cross-sectional area of the orbit in $\mathbf{k}$ space; $n$ is a large integer $(n>0) ; \gamma$ is a constant $(0 \leq \gamma<1)$, and $e$ is the electron charge. In what follows we shall consider only those orbits for which probabilities of intraband and interband magnetic breakdowns are negligible. In other words, the orbit under study does not come close to 


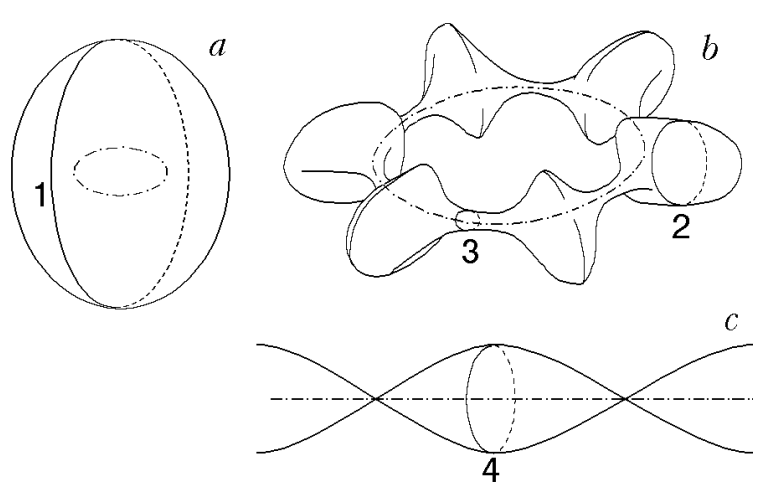

Fig. 1. The schematic sketch of Fermi surfaces for several metals with band-contact lines: the third-band electron «lens» of $\mathrm{Zn}$ and $\mathrm{Cd}(a)$; the second-band hole «coronet» («monster») of $\mathrm{Be}$ and $\mathrm{Mg}(b)$; the self-intersecting Fermi surface of graphite (c). The band-contact lines are shown as the dash-dot lines. The semiclassical orbits 3 and 4 link to the band-contact lines while the orbits 1 and 2 do not.

any other trajectory with the same $k_{z}$, and its shape differs noticeably from an intersecting one. In this case, according to Ref. 8 (see also Ref. 18), $\gamma$ always has the value

$$
\gamma=1 / 2 .
$$

It is this value that is commonly used in describing oscillation phenomena in metals [5] (e.g., the de Haas - van Alphen effect, the Shubnikov - de Haas effect etc.). If a magnetic breakdown occurs, $\gamma$ essentially depends on $\varepsilon$ and $k_{z}[10,11,15]$ but, as noted above, we shall not consider this situation.

In this paper we show that the equality

$$
\gamma=0
$$

can be valid if the closed electron orbit in $\mathbf{k}$ space associated with a certain energy band $\varepsilon_{0}(\mathbf{k})$ surrounds the line of degeneracy of this band with some other one. The above result depends neither on the form of $\varepsilon_{0}(\mathbf{k})$ in the neighborhood of the orbit nor on the shape and the size of this electron trajectory, and is topological in nature. It is due to the fact that the electron orbit links [19] (see Fig. 1) to the band-contact line which is the line of singularities for the Bloch wave functions. If the linking is absent, Eq. (2) holds. For the abovestated effect to be the case the band-contact line must satisfy the only condition: in its immediate vicinity the energies of the intersecting bands separate linearly in $\mathbf{k}$ as $\mathbf{k}$ moves away from the line. This condition is met for any accidental contact between the bands and in the case of degeneracy of the bands along a 3-fold symmetry axis of a crystal.
Equation (3) is obtainable from the results of Blount [20,21] and Roth [14,22]. In $\mathbf{k}$ space the effective one-band Hamiltonian of an electron in a magnetic field can be represented by a power series in $\mathbf{H}[21,22]$. Two terms of this series suffice to calculate $\gamma$ and thus we can use the following Hamiltonian:

$$
\hat{H}=\varepsilon_{0}(\hat{\mathbf{k}})+(e / c) H M_{0}(\hat{\mathbf{k}}),
$$

which must be considered as a symmetrized operator, that is, the components of $\hat{\mathbf{k}}$ always appear symmetrically in it. Here $\hat{\mathbf{k}}=\mathbf{k}-(e / \hbar c) \mathbf{A}\left(i \nabla_{\mathbf{k}}\right)$, $\mathbf{A}(\mathbf{r})$ is the vector potential for $\mathbf{H}$ and the quantity $M_{0}(\mathbf{k})$ is associated with the diagonal matrix element of the orbital angular momentum of the electron in the band under study (this band is designated by subscript 0 ). More precisely, $M_{0}$ is the periodic part of $z$-component of the above-mentioned element divided by the electron mass. The quantity $M_{0}$ falls into the intraband and interband constituents:

$$
M_{0}(\mathbf{k})=[\mathbf{v} \times \Omega]_{z}+\hbar \sum_{l \neq 0} \frac{\operatorname{Im}\left(\left(v_{x}\right)_{0 l}\left(v_{y}\right)_{0 l}^{*}\right)}{\varepsilon_{l}(\mathbf{k})-\varepsilon_{0}(\mathbf{k})},
$$

where $(\mathbf{v})_{0 l}$ is the interband matrix element of the velocity operator at the point $\mathbf{k}, \mathbf{v} \equiv(1 / \hbar) \nabla_{\mathbf{k}} \varepsilon_{0}(\mathbf{k})$, and $\Omega$ is the periodic part of the coordinate operator:

$$
\Omega(\mathbf{k})=i \int d \mathbf{r} u_{\mathbf{k} 0}^{*}(\mathbf{r}) \nabla_{\mathbf{k}} u_{\mathbf{k} 0}(\mathbf{r})
$$

Here the integration is carried out over a unit cell, and $u_{\mathbf{k} i}(\mathbf{r})$ denotes the periodic factor in the Bloch wave function of the band $l$ :

$$
\Psi_{\mathbf{k} l}(\mathbf{r})=\exp (i \mathbf{k r}) u_{\mathbf{k} l}(\mathbf{r}) .
$$

In the case of interest (the spin-orbit interaction is neglected for a crystal with inversion symmetry) $M_{0}(\mathbf{k})$ can be made to vanish at any point $\mathbf{k}$ of the Brillouin zone. This well-known statement results from the following considerations. Electron states are invariant under the transformation: $U=K I$, where $K$ and $I$ are the operators of complex conjugation and inversion, respectively. Hence, one can take the phases of Bloch factors in such a way as to fulfil the relation

$$
U u_{\mathbf{k} l}=u_{\mathbf{k} l}
$$

for any $l$. Under this condition the matrix elements $\mathbf{v}_{0 l}(\mathbf{k})$ are real and $\boldsymbol{\Omega}(\mathbf{k})=0$ [20]. Thus, Eq. (4) yields $M_{0}(\mathbf{k})=0$. To consider $M_{0}$ in the general 
situation when $u_{\mathbf{k} l}$ have arbitrary phases and do not satisfy condition (5) let us take the transformation

$$
u_{\mathbf{k} l} \rightarrow u_{\mathbf{k} l}^{\prime}=u_{\mathbf{k} l} \exp \left(i \varphi_{l}(\mathbf{k})\right),
$$

where $\varphi_{l}(\mathbf{k})$ are some regular functions of $\mathbf{k}$, and $u_{\mathbf{k} l}$ before the transformation obey Eq.(5) in a vicinity of the point concerned. Then

$$
\begin{gathered}
\mathbf{v}_{0 l} \rightarrow \mathbf{v}_{0 l}^{\prime}=\mathbf{v}_{0 l} \exp \left(i\left(\varphi_{l}-\varphi_{0}\right)\right), \\
\Omega \rightarrow \Omega^{\prime}=\Omega-\nabla_{\mathbf{k}} \varphi_{0}
\end{gathered}
$$

and we find from Eq. (4) that the interband part of $M_{0}$ is still equal to zero while its intraband component becomes nonvanishing and depends on the phase $\varphi_{0}$

According to Roth [14], $\gamma$ is determined by the formula

$$
\gamma-\frac{1}{2}=-\frac{1}{2 \pi} \oint_{\Gamma} \frac{M_{0}(\mathbf{k})}{v_{\perp}(\mathbf{k})} d \kappa,
$$

where $\Gamma$ is the closed semiclassical orbit in $\mathbf{k}$ space; $d \kappa$ is the length of an infinitesimal element of $\Gamma$ : $v_{\perp}$ is the absolute value of the projection of $\mathbf{v}$ on the plane of the orbit. Taking into account that the interband part of $M_{0}$ is zero, Eq.(9) can be rearranged as follows:

$$
\gamma-\frac{1}{2}=-\frac{1}{2 \pi} \oint \Omega d \mathbf{k},
$$

where $d \mathbf{k} \equiv d \kappa\left[\mathbf{i}_{z} \times \mathbf{v}\right] / v_{\perp}$ and $\mathbf{i}_{z}$ is the unit vector parallel to $\mathbf{H}$ ( $d \mathbf{k}$ is aligned with the tangent to $\Gamma$ and $|d \mathbf{k}|=d \kappa)$. It is evident from Eqs. (8) and (10) that, in contrast to $M_{0}$, the measurable quantity $\gamma$ is invariant under transformation (6).

It is generally believed that $\Omega$ (and $M_{0}$ ) can be made to vanish everywhere over the Brillouin zone, and thus $\gamma=1 / 2$. This does be true in the absence of the degeneracy. However, if a line of the contact between the band under study and some other one exists, and the energies of the bands separate linearly in $\mathbf{k}$ in the vicinity of the line, then, according to Blount [23], $\Omega$ can be made to vanish locally (i.e., in the neighborhood of any point that does not lie in the band-contact line) but this is impossible to attain along the whole length of a closed path $P$ surrounding the line [24]. Moreover, one has

$$
\oint_{P} \Omega d \mathbf{k}= \pm \pi
$$

where the sign in the right-hand side of the equation is determined by a direction of the integration. We emphasize that the integral in Eq.(11) does not depend on the shape and the size of the contour $P$. This is not surprising, since the equation

$$
\nabla_{\mathbf{k}} \times \Omega(\mathbf{k})=0
$$

holds everywhere out of the band-contact line [25]. Finally, one more comment needs to be made. In general, the term $2 \pi q$ must be added to the righthand side of Eq.(11) [23] where $q$ is some integer. However it can be shown [3] that $q=0$ when the spin-orbit interaction is taken into account. (Besides, nonzero $q$ would modify $n$ and not affect $\gamma$.)

Now we are able to find $\gamma$ for any mutual arrangement of the semiclassical electron orbit $\Gamma$ and the band-contact line. If $\Gamma$ links to this line (see, e.g., orbits 3 and 4 in Fig.1) formula (3) follows from Eqs. (10),(11) (note that the values $\gamma=1$ and $\gamma=0$ are equivalent). If the linking is absent, a surface with boundary $\Gamma$ necessarily exists which does not intersect the band-contact line [19] (for the surface in the case of orbit 1 (or 2) we can take a part of the constant-energy one shown in Fig. 1). Transforming Eq.(10) into the integral over this surface and taking into account Eq.(12), we arrive at formula (2). Interestingly, Eq.(2) is also obtained when $\Gamma$ links to an even number of band-contact lines (such a situation takes place, e.g., for any central cross-section of the second-band Fermi surface of $\mathrm{Al}$ ).

In the cases of Eqs.(2) and (3) the appropriate sets of the magnetic energy levels are shifted relative each other. The origin of this shift is easy to understand. As discussed above, the quantity $M_{0}$ can be made to vanish for any nondegenerate electron state with a fixed wave vector $\mathbf{k}$. In essence, this is the so-called quenching of orbital angular momenta [26] (but only their periodic parts are quenched in the case of nonlocalized states considered here). Then Eq. (10) must be interpreted as the lack of the quenching for the semiclassical electron moving round the band-contact line. This gives rise to the additional magnetic moment of the electron: $\left(|e| \hbar / 2 m^{*} c\right)$, where $m^{*}$ is its cyclotron mass:

$$
m^{*}=\frac{\hbar^{2}}{2 \pi}\left(\frac{\partial S}{\partial \varepsilon}\right)=\frac{\hbar}{2 \pi} \oint_{\Gamma} \frac{d \kappa}{v_{\perp}} .
$$

It is the interaction of this moment with $\mathbf{H}$ that leads to the above-mentioned shift of the magnetic energy levels.

The obtained result is closely analogous to the Aharonov-Bohm effect [27]. As pointed out by 
Blount [20], the quantity $\Omega$ is similar to a vector potential for a magnetic field [see Eqs. (6), (8)]. Taking Eqs. (11), (12) into account, we can treat a band-contact line as an infinitely thin «solenoid» which carries the fixed flux of the «field» $\left[\nabla_{k} \times \Omega\right]$. With this in mind the above-mentioned analogy becomes apparent. Although the semiclassical electron moving round the band-contact line does not reach the region in which the «field» is nonzero, it experiences the «vector potential» $\Omega$ that cannot be made to vanish along the whole length of the orbit. The semiclassical electron state with energy determined by Eq. (1) is the standing wave. If the electron orbit surrounds the band-contact line (i.e., the «solenoid»), the interference picture corresponding to this wave is shifted as compared to the case when the line is absent. This shift manifests itself as the change in $\gamma$.

The above results can be also understood with the concept of Berry's phase [28]. If the Hamiltonian of a quantum system depends on parameters and the parameters undergo adiabatic changes so that they eventually return to their original values, then the wave function of the system can acquire, according to Berry, some constant phase in addition to the familiar dynamical one. This additional phase is completely determined by a closed trajectory $P$ of the system in the parameter space and does not depend on details of the temporal evolution. In addition, it was obtained [28] that the phase is equal to $\pi$ when the trajectory $P$ surrounds a point of degeneracy of the Hamiltonian. More recently, Zak [29] has argued that Berry's results are applicable to an electron moving in a crystal, with $\mathbf{k}$ space playing the role of the parameter space, and the above-mentioned phase is described by the integral given in the left-hand side of Eq. (11). Then Eq. (3) may be interpreted as a manifestation of Berry's phase. In this connection we emphasize that measurements of $\gamma$ in crystals with band-contact lines offer a way of detecting this phase in physics of metals [30].

The value $\gamma$ can be experimentally determined through the investigation of oscillation effects in metals [5]. Since the measurement of $\gamma$ is easiest to make for semiclassical orbits corresponding to small extremal cross sections of a Fermi surface, we point out that such orbits exist, e.g., in beryllium, magnesium, graphite, and in these metals they link to the band-contact lines (see Fig. 1). In Be and $\mathrm{Mg}$ the accidental contact between the second and third bands occurs in the basal plane of the crystals. If $\mathbf{H}$ lies, e.g. , in this plane too, Eq. (3) must be valid for the orbits on the «necks» of the second-band hole «coronet» (or «monster»). It should be noted that in $\mathrm{Zn}$ and $\mathrm{Cd}$ which are akin, in many respects, to $\mathrm{Be}$ and $\mathrm{Mg}$ the same band-contact line is located in the third-band electron «lens» and does not link to the semiclassical orbits (therefore, in this case $\gamma=1 / 2$ ). In graphite the degeneracy of two bands takes place along the vertical edge $\mathrm{HKH}$ of the Brillouin zone (i.e., along the 3 -fold symmetry axis). Thus, Eq. (3) is expected to be true for the extremal orbit surrounding the point $K$ (see orbit 4 in Fig. 1).

In summary, we have shown that in quantization condition (1) $\gamma$ is equal to zero when the appropriate semiclassical orbit of an electron links to the band-contact line. This value differs essentially from the conventional one $\gamma=1 / 2$. Thus, measurements of $\gamma$ can provide a possibility of detecting band-contact lines in metals (beryllium, magnesium and graphite appear to have considerable promise on this point).

1. C. Herring, Phys. Rev. 52, 365 (1937).

2. D. A. Papaconstantopoulos, Handbook of the Band Structure of Elemental Solids, Plenum, New York (1986).

3. G. P. Mikitik and Yu. V. Sharlai, Zh. Eksp. Teor. Fiz. 114, 1375 (1998) [ JETP 87, 747 (1998)].

4. I. M. Lifshitz, M. Ya. Azbel', and M. I. Kaganov, Electron Theory of Metals, Consultant Bureau, New York (1973).

5. D. Shoenberg, Magnetic Oscillations in Metals, Cambridge Univ. Press, Cambridge-Sydney (1984).

6. L. Onsager, Phil. Mag. 43, 1006 (1952).

7. I. M. Lifshitz and A. M. Kosevich, Zh. Eksp. Teor. Fiz. 29, 730 (1955) [Sov. Phys. JETP 2, 636 (1956)].

8. G. E. Zilberman, Zh. Eksp. Teor. Fiz. 32, 296 (1957) [Sov. Phys. JETP 5, 208 (1957)].

9. G. E. Zilberman, Zh. Eksp. Teor. Fiz. 33, 387 (1957) [Sov. Phys. JETP 6, 299 (1958)].

10. G. E. Zilberman, Zh. Eksp. Teor. Fiz. 34, 748 (1958) [Sov. Phys. JETP 7, 513 (1958)].

11. M. Ya. Azbel', Zh. Eksp. Teor. Fiz. 39, 1276 (1960) [Sov. Phys. JETP 12, 891 (1961)].

12. M. Ya. Azbel', Zh. Eksp. Teor. Fiz. 46, 929 (1964) [Sov. Phys. JETP 19, 634 (1964)].

13. L. A. Falkovsky, Zh. Eksp. Teor. Fiz. 49, 609 (1965) [Sov. Phys. JETP 22, 423 (1966)].

14. L. M. Roth, Phys. Rev. 145, 434 (1966).

15. A. A. Slutskin, Zh. Eksp. Teor. Fiz. 53, 767 (1967) [Sov. Phys. JETP 26, 474 (1968)].

16. S. S. Nedorezov, Fiz. Nizk. Temp. 2, 1047 (1976) [Sov. Phys. Low Temp. Fiz. 2, 513 (1976)].

17, B. M. Gorbovitsky and V. I. Perel', Zh. Eksp. Teor. Fiz. 85, 1812 (1983) [Sov. Phys. JETP 58, 1054 (1983)].

18. E. M. Lifshitz and L. P. Pitaevskii, Statistical Physics, Permagon, Oxford (1986), vol. 9.

19. B. A. Dubrovin, S. P. Novikov, and A. T. Fomenko, Modern Geometry, Nauka, Moscow (1979).

20. E. I. Blount, Solid State Physics, Academic Press, New York-London (1962), vol. 13

21. E. I. Blount, Phys. Rev. 126, 1636 (1962).

22. L. M. Roth, J. Phys. Chem. Solids 23, 433 (1962).

23. See pages 327, 328, 361 in Ref. 20. 
24. This result is due to the nonanalytical behavior of $u_{\mathrm{k} 0}$ in the band-contact line and can be explained in the following manner. If the point $\mathbf{k}$ moves along $P$ and in the process the phases of $u_{\mathrm{k} 0}$ are chosen so that condition (5) be met, then $u_{\mathrm{k} 0}$ goes over into $-u_{\mathrm{k} 0}$ when $\mathbf{k}$ returns to the starting position $\mathbf{k}_{0}$. Thus, continuous $u_{\mathrm{k} 0}$ cannot satisfy condition (5) along the whole length of $P$.

25. Since $\nabla_{\mathrm{k}} \times \Omega$ is invariant under phase transformations and $\Omega$ can be made to vanish locally, Eq. (12) is true for any choice of $u_{\mathrm{k} 0}$.
26. R. W. White, Quantum Theory of Magnetism, SpringerVerlag, Berlin-New York (1983).

27. Y. Aharonov and D. Bohm, Phys. Rev. 115, 485 (1959).

28. M. V. Berry, Proc. Roy. Soc., London A392, 45 (1984).

29. J. Zak, Phys. Rev. Lett. 62, 2747 (1989).

30. As to experimental investigations of Berry' phase in molecular physics, optics and resonance phenomena in nuclear quadrupole systems, see, e.g., papers: G. Delacretaz et al., Phys. Rev. Lett. 56, 2598 (1986); A. Tomita and R. Y. Chiao, Phys. Rev. Lett. 57, 937 (1986); R. Tycko, Phys. Rev. Lett. 58, 2281 (1987). 